\title{
QUEEN'S
UNIVERSITY
BELFAST
}

\section{On functions of bounded variation}

Aistleitner, C., Pausinger, F., Svane, A. M., \& Tichy, R. F. (2017). On functions of bounded variation. Mathematical Proceedings of the Cambridge Philosophical Society, 162(3), 405-418.

https://doi.org/10.1017/S0305004116000633

\section{Published in:}

Mathematical Proceedings of the Cambridge Philosophical Society

\section{Document Version:}

Peer reviewed version

\section{Queen's University Belfast - Research Portal:}

Link to publication record in Queen's University Belfast Research Portal

\section{Publisher rights}

Copyright 2017 Cambridge University Press. This work is made available online in accordance with the publisher's policies. Please refer to any applicable terms of use of the publisher.

\section{General rights}

Copyright for the publications made accessible via the Queen's University Belfast Research Portal is retained by the author(s) and / or other copyright owners and it is a condition of accessing these publications that users recognise and abide by the legal requirements associated with these rights.

\section{Take down policy}

The Research Portal is Queen's institutional repository that provides access to Queen's research output. Every effort has been made to ensure that content in the Research Portal does not infringe any person's rights, or applicable UK laws. If you discover content in the Research Portal that you believe breaches copyright or violates any law, please contact openaccess@qub.ac.uk. 


\title{
On functions of bounded variation $\dagger$
}

\author{
By CHRISTOPH AISTLEITNER \\ Johannes Kepler University Linz, \\ E-Mail: aistleitner@math.tugraz.at, \\ AND FLORIAN PAUSINGER \\ TU Munich, \\ E-Mail: florian.pausinger@gmx.at \\ AND ANNE MARIE SVANE \\ Aarhus University, \\ E-Mail: amsvane@math.au.dk \\ AND ROBERT F. TICHY \\ TU Graz, \\ E-Mail: tichy@tugraz.at
} (Received ) trie und Visualisierung, Boltzmannstr. 3, 85748 Garching. 
Abstract

The recently introduced concept of $\mathcal{D}$-variation unifies previous concepts of variation of multivariate functions. In this paper, we give an affirmative answer to the open question from $[\mathbf{2 0}]$ whether every function of bounded Hardy-Krause variation is Borel measurable and has bounded $\mathcal{D}$-variation. Moreover, we show that the space of functions of bounded $\mathcal{D}$-variation can be turned into a commutative Banach algebra.

Keywords: Harman variation, Hardy-Krause variation, Koksma-Hlawka theorem, bounded variation.

MSC2010: 26B30; 65D30 11K38.

\section{Contents}

1 Introduction 2

2 Different notions of variation 3

3 Borel measurability of functions of bounded Hardy-Krause variation 6

4 Equivalence of Hardy-Krause and $\mathcal{R}^{*}$-variation 9

$5 \quad$ Algebraic structure of $\mathcal{V}(\mathcal{D}) \quad 13$

References $\quad 15$

\section{Introduction}

It is a classical problem to generalise the notion of total variation of a one-dimensional function to multivariate functions and study conditions under which a function has bounded variation. The algebraic properties of the corresponding spaces of functions of bounded variation are of particular interest in numerical integration. Let $f$ be a realvalued measureable function over a compact Hausdorff space $X$, equipped with a sigmafield $\mathcal{F}$ and a normalized measure $\mu$. Furthermore, let $x_{1}, x_{2} \ldots, x_{N} \in X$. The famous Koksma-Hlawka inequality is a general principle to bound the approximation error

$$
\left|\frac{1}{N} \sum_{j=1}^{N} f\left(x_{j}\right)-\int_{X} f(x) d \mu(x)\right|
$$

by the product of two independent factors. One of these factor depends only on the function $f$ (more precisely, on the variation of $f$ ) and the other factor depends only on the discrete point set (the discrepancy of $x_{1}, x_{2}, \ldots, x_{N}$ ). Informally speaking, the discrepancy measures the deviation between the empirical distribution of the points $x_{1}, \ldots, x_{N}$ and the measure $\mu$. The classical setting is that of $X$ being the $d$-dimensional unit cube $[0,1]^{d}$ and $\mu$ being the $d$-dimensional Lebesgue measure; in this setting, the KoksmaHlawka inequality reads as

$$
\left|\frac{1}{N} \sum_{j=1}^{N} f\left(\mathbf{x}_{j}\right)-\int_{[0,1]^{d}} f(\mathbf{x}) d \mathbf{x}\right| \leq D_{N}^{*}\left(\mathbf{x}_{1}, \ldots, \mathbf{x}_{N}\right) \cdot \operatorname{Var}_{\mathrm{HK}} f,
$$

where $D_{N}^{*}$ is the so-called star discrepancy and $\operatorname{Var}_{\mathrm{HK}}$ is the variation in the sense of Hardy and Krause; for details on this basic result of discrepancy theory, see for example $[\mathbf{9}, 17]$. 
It is well known that the space of all real-valued functions of bounded total variation on the compact interval $[a, b]$ is a commutative Banach algebra with respect to pointwise multiplication. However, it is not obvious how to generalize this notion of bounded variation to the case of multivariate functions. Hardy [11] and Krause [16] introduced a concept of bounded variation for multivariate functions, which was used by Hlawka [13] to generalize the one-dimensional Koksma inequality [15] and to obtain the classical version of the Koksma-Hlawka inequality as stated in (1·2). Götz [10] proved a version of the Koksma-Hlawka inequality for general measures (rather than only Lebesgue measure), and recently, Brandolini, Colzani, Gigante and Travaglini $[6,7]$ replaced the integration domain $[0,1]^{s}$ by an arbitrary bounded Borel subset of $\mathbb{R}^{d}$ and proved the inequality for piecewise smooth integrands.

The notion of Hardy-Krause variation was generalised in a natural way by Blümlinger and Tichy [4], who proved that the corresponding space of functions of bounded variation is a commutative Banach algebra. However, especially in the context of numerical integration, these different notions of Hardy-Krause variation come with the severe drawback that many functions of practical interest have unbounded variation (e.g. the indicator function of a ball or a tilted box). Recently Harman [12] introduced a new notion of variation, which remains finite for certain discontinuous functions with unbounded variation in the sense of Hardy and Krause, and proved a Koksma-Hlawka inequality in this settig. Unfortunately, the space of functions of bounded Harman variation lacks many of the nice algebraic properties of the Hardy-Krause variation.

This was the motivation that led to the introduction of a general framework of variations in [20]. The concept of $\mathcal{D}$-variation unifies the different notions of variation and is not restricted to integrals over $[0,1]^{d}$, but works for integrals over arbitrary compact Hausdorff spaces. It was shown to coincide with Hardy-Krause variation in special cases. The first aim of our paper is to show that every function of bounded Hardy-Krause variation also has bounded variation in the new sense, thus answering a question which was left open in [20]; see Section 4. In particular this also means that every function of bounded Hardy-Krause variation is Borel measurable. This is a fundamental result which we did not find anywhere in the literature, for which reason we also provide a self-contained proof in Section 3.

Functions of bounded Hardy-Krause variation received a lot of attention in the literature; see $[\mathbf{1}, \mathbf{2}, \mathbf{4}, \mathbf{5}, \mathbf{8}, \mathbf{1 8}]$. Given our results, it is natural to ask whether results about the structure of the space of functions of bounded Hardy-Krause variation, e.g., that it is a Banach algebra (see $[\mathbf{4}, \mathbf{5}]$ ), also extend to our more general notion. We discuss this question in Section 5, where we show that the space of functions of bounded $\mathcal{D}$-variation is indeed a commutative Banach algebra.

\section{Different notions of variation}

In the following we introduce the two definitions of variation of a multivariate function that we consider: the classical Hardy-Krause variation and the recently introduced $\mathcal{D}$ variation. 


\section{2·1. Hardy-Krause variation}

Definition. In the following, we use the notation of Owen [19]. Let $f(\mathbf{x})$ be a function on $[0,1]^{d}$. If $\mathbf{a}=\left(a_{1}, \ldots, a_{d}\right)$ and $\mathbf{b}=\left(b_{1}, \ldots, b_{d}\right)$ are elements of $[0,1]^{d}$ such that $a_{i} \leq b_{i}\left(a_{i}<b_{i}\right)$ for all $1 \leq i \leq d$, then we write $\mathbf{a} \leq \mathbf{b}(\mathbf{a}<\mathbf{b})$. For $u \subseteq\{1, \ldots, d\}$, we denote by $\mathbf{a}^{u}: \mathbf{b}^{-u}$ the point with $i$-th coordinate equal to $a_{i}$ if $i \in u$ and equal to $b_{i}$ otherwise. The set $-u$ is the set complement of $u$ in $\{1, \ldots, d\}$. Using this notation, we introduce the $d$-dimensional difference operator

$$
\Delta^{(d)}(f ; R)=\Delta(f ; R)=\sum_{u \subseteq\{1, \ldots, d\}}(-1)^{|u|} f\left(\mathbf{a}^{u}: \mathbf{b}^{-u}\right),
$$

which assigns to the axis-parallel rectangle $R=[\mathbf{a}, \mathbf{b}]$ a $d$-dimensional quasi-volume.

In dimension $d=1$, a ladder $\mathcal{Y}$ on the interval $[0,1]$ is a partition of $[0,1]$, i.e. a sequence $0=y_{1}<\cdots<y_{k}<1$. A ladder in $[0,1]^{d}$ is a set of the form $\mathcal{Y}=\prod_{j=1}^{d} \mathcal{Y}^{j} \subseteq[0,1]^{d}$, where each $\mathcal{Y}^{j}$ is a one-dimensional ladder. Let $\mathbb{Y}$ be the set of all ladders on $[0,1]^{d}$. Suppose $\mathcal{Y}^{j}=\left\{y_{1}^{j}<\cdots<y_{k_{j}}^{j}\right\}$. Define the successor $\left(y_{i}^{j}\right)_{+}$of $y_{i}^{j}$ to be $y_{i+1}^{j}$ if $i<k_{j}$ and $\left(y_{k_{j}}^{j}\right)_{+}=1$. If $\mathbf{y}=\left(y_{i_{1}}^{1}, \ldots, y_{i_{d}}^{d}\right) \in \mathcal{Y}$, then we define its successor to be $\mathbf{y}_{+}=$ $\left(\left(y_{i_{1}}^{1}\right)_{+}, \ldots,\left(y_{i_{d}}^{d}\right)_{+}\right)$. For a ladder $\mathcal{Y}$ in $[0,1]^{d}$, we have by [19, Proposition 2]

$$
\Delta\left(f ;[0,1]^{d}\right)=\sum_{\mathbf{y} \in \mathcal{Y}} \Delta\left(f ;\left[\mathbf{y}, \mathbf{y}_{+}\right]\right) .
$$

Define the variation over $\mathcal{Y}$ by

$$
V_{\mathcal{Y}}\left(f ;[0,1]^{d}\right)=\sum_{\mathbf{y} \in \mathcal{Y}}\left|\Delta\left(f ;\left[\mathbf{y}, \mathbf{y}_{+}\right]\right)\right| .
$$

Then the Vitali variation of $f$ over $[0,1]^{d}$ is defined by

$$
V\left(f ;[0,1]^{d}\right)=\sup _{\mathcal{Y} \in \mathbb{Y}} V_{\mathcal{Y}}\left(f ;[0,1]^{d}\right) .
$$

For a subset $u \subseteq\{1, \ldots, d\}$, let

$$
\Delta_{u}(f ;[\mathbf{a}, \mathbf{b}])=\sum_{v \subseteq u}(-1)^{|v|} f\left(\mathbf{a}^{v}: \mathbf{b}^{-v}\right) .
$$

Let $\mathbf{0}=(0, \ldots, 0) \in[0,1]^{d}$ and $\mathbf{1}=(1, \ldots, 1) \in[0,1]^{d}$. Given a ladder $\mathcal{Y}$, there is a corresponding ladder $\mathcal{Y}_{u}=\left\{\mathbf{y}^{u}: \mathbf{1}^{-u} \mid \mathbf{y} \in \mathcal{Y}\right\}$ on the $|u|$-dimensional face of $[0,1]^{d}$ consisting of points of the form $\mathbf{x}^{u}: \mathbf{1}^{-u}$ (we interpret $\mathcal{Y}_{\emptyset}$ as $\{\mathbf{1}\}$ ). The operation of the successor is also defined on $\mathcal{Y}_{u}$, and again we have,

$$
\Delta_{u}\left(f ;[0,1]^{d}\right)=\sum_{\mathbf{y} \in \mathcal{Y}_{u}} \Delta_{u}\left(f ;\left[\mathbf{y}, \mathbf{y}_{+}\right]\right) .
$$

Furthermore, we define

$$
V_{\mathcal{Y}_{u}}\left(f ;[0,1]^{d}\right)=\sum_{\mathbf{y} \in \mathcal{Y}_{u}}\left|\Delta\left(f ;\left[\mathbf{y}, \mathbf{y}_{+}\right]\right)\right|,
$$

which is the variation over the ladder $\mathcal{Y}_{u}$ of the restriction of $f$ to the face of $[0,1]^{d}$ specified by $u$. The Hardy-Krause variation is defined as

$$
H K\left(f ;[0,1]^{d}\right)=\sum_{\emptyset \neq u \subseteq\{1, \ldots, d\}} \sup _{\mathcal{Y} \in \mathbb{Y}} V_{\mathcal{Y}_{u}}\left(f ;[0,1]^{d}\right) .
$$


$\mathcal{H} \mathcal{K}$ denotes the class of functions with bounded Hardy-Krause variation. In words, the Hardy-Krause variation is the sum of the Vitali variations of all the restrictions of $f$ to those faces of $[0,1]^{d}$ adjecent to $\mathbf{1}$.

Leonov's result. We follow $[\mathbf{2}]$ and call a function $f:[0,1]^{d} \rightarrow \mathbb{R}$ completely monotone if the restriction $f_{\mid R}$ of $f$ to any axis-parallel box $R=[\mathbf{a}, \mathbf{b}] \subseteq[0,1]^{d}$ of dimension $1 \leq s \leq d$ with $\mathbf{a} \leq \mathbf{b}$ satisfies $\Delta^{(s)}\left(f_{\mid R},[\mathbf{a}, \mathbf{b}]\right) \geq 0$. The $s$ in $\Delta^{(s)}$ is the dimension of $[\mathbf{a}, \mathbf{b}]$ and marks that $f_{\mid R}$ is considered as a function of $s$ variables when computing $\Delta^{(s)}$. We shall need the following result by Leonov [18]:

Lemma 2·1 (Leonov [18]). Any function of bounded Hardy-Krause variation can be written as the difference of two completely monotone functions.

\subsection{D-variation}

In the following, we recall the notion of variation introduced in $[\mathbf{2 0}]$. Let $\mathcal{D}$ denote an arbitrary family of measurable subsets of $[0,1]^{d}$ with $\emptyset,[0,1]^{d} \in \mathcal{D}$. Let $\mathcal{S}(\mathcal{D})$ denote the corresponding vector space of simple functions

$$
f=\sum_{i=1}^{m} \alpha_{i} \mathbb{1}_{A_{i}}
$$

where $\alpha_{i} \in \mathbb{R}, A_{i} \in \mathcal{D}$, and $m \in \mathbb{N}$. Note that the representation of $f$ is of course not unique. We say that a set $A \subseteq[0,1]^{d}$ is an algebraic sum of sets in $\mathcal{D}$ if there exist $A_{1}, \ldots, A_{m} \in \mathcal{D}$ such that

$$
\mathbb{1}_{A}=\sum_{i=1}^{n} \mathbb{1}_{A_{i}}-\sum_{i=n+1}^{m} \mathbb{1}_{A_{i}}
$$

and we define $\mathcal{A}$ to be the collection of algebraic sums of sets in $\mathcal{D}$.

Inspired by [12], we define the Harman complexity $h(A)$ of a set $A \in \mathcal{A}$ with $A \neq[0,1]^{d}$ and $A \neq \emptyset$, as the minimal number $m$ such that there exists $A_{1}, \ldots, A_{m}$ with

$$
\mathbb{1}_{A}=\sum_{i=1}^{n} \mathbb{1}_{A_{i}}-\sum_{i=n+1}^{m} \mathbb{1}_{A_{i}}
$$

for some $n \in\{0, \ldots, m\}$ and either $A_{i} \in \mathcal{D}$ or $[0,1]^{d} \backslash A_{i} \in \mathcal{D}$. Moreover, we define $h\left([0,1]^{d}\right)=h(\emptyset)=0$.

The definition of variation is given in two steps. First, for $f \in \mathcal{S}(\mathcal{D})$, we define

$$
V_{\mathcal{S}, \mathcal{D}}(f):=\inf \left\{\sum_{i=1}^{m}\left|\alpha_{i}\right| h_{\mathcal{D}}\left(A_{i}\right) \quad \mid f=\sum_{i=1}^{m} \alpha_{i} \mathbb{1}_{A_{i}}, \quad \alpha_{i} \in \mathbb{R}, A_{i} \in \mathcal{D}\right\} .
$$

Second, let $\mathcal{V}_{\infty}(\mathcal{D})$ be the collection of all measurable functions $f:[0,1]^{d} \rightarrow \mathbb{R}$ for which there exists a sequence of $f_{i} \in \mathcal{S}(\mathcal{D})$ that converges to $f$ in the supremum norm $|\cdot|_{\infty}$.

Definition $2 \cdot 2\left([\mathbf{2 0}]\right.$, Definition 3.2). We define the $\mathcal{D}$-variation of $f \in \mathcal{V}_{\infty}(\mathcal{D})$ as

$$
V_{\mathcal{D}}(f)=\inf \left\{\liminf _{i} V_{\mathcal{S}, \mathcal{D}}\left(f_{i}\right) \quad\left|\quad f_{i} \in \mathcal{S}(\mathcal{D}), \lim _{i}\right| f-\left.f_{i}\right|_{\infty}=0\right\}
$$

and set $V_{\mathcal{D}}(f)=\infty$ if $f \notin \mathcal{V}_{\infty}(\mathcal{D})$. The space of functions of bounded $\mathcal{D}$-variation is 
denoted by

$$
\mathcal{V}(\mathcal{D})=\left\{f \in \mathcal{V}_{\infty}(\mathcal{D}) \quad \mid \quad V_{\mathcal{D}}(f)<\infty\right\}
$$

Among the classes of sets $\mathcal{D}$ which are of particular interest are the class $\mathcal{K}$ of convex sets and the class $\mathcal{R}^{*}$ of axis parallel boxes containing $\mathbf{0}$ as a vertex. In the following we recall the most important properties of this notion.

Proposition $2 \cdot 3$.

(i) $\mathcal{V}_{\infty}(\mathcal{D})$ and $\mathcal{V}(\mathcal{D})$ are vector spaces. In particular, $V_{\mathcal{D}}$ defines a semi-norm on $\mathcal{V}(\mathcal{D})$. (ii) $\mathcal{V}_{\infty}(\mathcal{D})$ is closed under limits in the supremum-norm. We have the following lower semi-continuity: if $\left|f-f_{i}\right|_{\infty} \rightarrow 0$ then $V_{\mathcal{D}}(f) \leq \liminf _{i} V_{\mathcal{D}}\left(f_{i}\right)$.

(iii) If $\mathcal{D}$ is closed under intersection, then $\mathcal{V}(\mathcal{D})$ is closed under multiplication, and

$$
V_{\mathcal{D}}(f g) \leq 3 V_{\mathcal{D}}(f) V_{\mathcal{D}}(g)+\inf |f| V_{\mathcal{D}}(g)+\inf |g| V_{\mathcal{D}}(f) .
$$

Proof. (i) is [20, Proposition 3.5], (ii) is [20, Proposition 3.6], (iii) is [20, Theorem 3.7].

\section{Borel measurability of functions of bounded Hardy-Krause variation}

The aim of this section is to give an independent proof that every function of bounded Hardy-Krause variation is Borel measurable. This fact also plays a key role in the equivalence of Hardy-Krause variation and $\mathcal{R}^{*}$-variation, which will be stated and proved in the subsequent section.

THEOREM 3.1. Every function of bounded Hardy-Krause variation is Borel measurable. More precisely, every real-valued function on $[0,1]^{d}$ which has bounded HK-variation is $\left([0,1]^{d}, \mathcal{B}\left([0,1]^{d}\right)\right)-(\mathbb{R}, \mathcal{B}(\mathbb{R}))$-measurable.

We have looked in the literature very carefully, but have not found anywhere the fact that finite HK-variation implies Borel measurability. It is remarkable that such a fundamental property of functions of bounded HK-variation has not been investigated before. However, the proof is far from being trivial (see below). Recall from Lemma $2 \cdot 1$ that a function of bounded HK-variation decomposes into a difference of two completely monotone functions (see also [2]), so the assertion of Theorem $3 \cdot 1$ follows from a similar result for completely monotone functions, stated in Theorem 3.2 below. The fact that completely monotone functions are Borel measurable also seems to be new. Note that coordinatewise monotonicity is not sufficient for a multivariate function to be Borel measurable. For a two-dimensional counterexample, define $f(x, y)=0$ for $x+y<1$, $f(x, y)=1$ for $x+y>1$, and for $x+y=1$ set $f(x, y)=1 / 2$ for $x \in E$ and $f(x, y)=0$ otherwise, where $E \subset[0,1]$ is not Borel measurable. Then $f^{-1}(\{1 / 2\})$ is not in $\mathcal{B}\left([0,1]^{2}\right)$.

On the other hand, coordinatewise monotonicity is actually sufficient for Lebesgue measurability of a multivariate function. That means, a function which is coordinatewise monotone is $\left([0,1]^{d}, \mathcal{L}\left([0,1]^{d}\right)\right)-(\mathbb{R}, \mathcal{B}(\mathbb{R}))$-measurable, where $\mathcal{L}$ is the Lebesgue sigmafield (the completion of the Borel sigma-field). A possible proof goes as follows. We use induction on $d$. The case $d=1$ is trivial. Now let $f$ be a function of $d$ variables which is increasing in every coordinate. For fixed $a \in \mathbb{R}$, define $g\left(x_{1}, \ldots, x_{d-1}\right)=\sup \{y \in$ $\left.[0,1]: f\left(x_{1}, \ldots, x_{d-1}, y\right) \leq a\right\}$, where the supremum of the empty set is understood to be zero. Then $g$ is monotonic decreasing, and, by the induction hypothesis, Lebesgue 
measurable. Thus the set $A=\left\{\left(x_{1}, \ldots, x_{d-1}, y\right): g\left(x_{1}, \ldots, x_{d-1}\right)<y\right\}$ is also Lebesgue measurable. Moreover, the set $B=\left\{\left(x_{1}, \ldots, x_{d-1}, y\right): g\left(x_{1}, \ldots, x_{d-1}\right)=y\right\}$ is of measure zero (by Fubini's theorem). Now the set $\{f>a\}$ differs from $A$ only by a subset of $B$, which has measure zero, and hence is Lebesgue measurable.

The remarks above show that these measurability issues are rather delicate, and should be treated very carefully (as a deterrent example cf. [14], where it is proved that every multivariate, coordinatewise monotonic function is "measurable", without any mention in the whole paper which kind of measurability is actually meant - in fact the author talks about Lebesgue measurability, but careless readers may easily be misled).

THEOREM 3.2. Every completely monotone and real-valued function on $[0,1]^{d}$ is $\left([0,1]^{d}, \mathcal{B}\left([0,1]^{d}\right)\right)-(\mathbb{R}, \mathcal{B}(\mathbb{R}))$-measurable.

Proof of Theorems $3 \cdot 1$ and $3 \cdot 2$. By Lemma 2.1, a function of bounded HK-variation can be written as the difference of two completely monotone functions. Thus Theorem $3 \cdot 1$ is a consequence of Theorem $3 \cdot 2$, and in the sequel we will assume that $f$ is a completely monotone function.

We proceed by induction on the number of variables $d$. In the case $d=1$ complete monotonicity reduces to (ordinary) monotonicity, and the Borel measurability of monotonic functions in one variable is a classical result. This proves the initial step of the induction.

Now we assume that the induction hypothesis holds for all completely monotone functions which have less than $d$ variables, and assume that $f$ is a completely monotone function on $[0,1]^{d}$. It is a well-known fact that all the discontinuities of a completely monotone function lie on an at most countable set of hyperplanes of dimensions $d-1$, all of which are parallel to the coordinate axes (this fact was probably first noted by Young and Young $[\mathbf{2 2}]$, and rediscovered by Antosik [3]). As a consequence, roughly speaking, $f$ decomposes into a continuous part (which is measurable by continuity) and into countably many lower-dimensional functions (which are measurable by the induction hypothesis). However, this argument has to be carried out very carefully; all the details are given below.

We write $H_{1}, H_{2}, \ldots$ for the collection of $(d-1)$-dimensional hyperplanes where the discontinuities of $f$ are situated, and we set

$$
D=[0,1]^{d} \backslash\left(\bigcup_{k=1}^{\infty} H_{k}\right) .
$$

Since $f$ is assumed to be completely monotone, by definition it is monotonically increasing. Thus there exists a number $m$ such that $m<f(\mathbf{0}) \leq f(\mathbf{x})$ for all $\mathbf{x} \in[0,1]^{d}$. For $k \geq 1$, we define

$$
f_{k}(\mathbf{x})= \begin{cases}f(\mathbf{x}) & \text { for } \mathbf{x} \in H_{k}, \\ m & \text { otherwise }\end{cases}
$$

Let $k$ be given. Then there exist an index $i \in\{1, \ldots, d\}$ and a number $a \in[0,1]$ such that the hyperplane $H_{k}$ consists of all the points $\left\{\mathbf{x}=\left(x^{(1)}, \ldots, x^{(d)}\right) \in[0,1]^{d}: x^{(i)}=a\right\}$. Furthermore, the $d$-variate function $f_{k}$ induces in a natural way a $d$-1-variate function 
$\hat{f}_{k}$ by the relation

$$
\begin{aligned}
& \hat{f}_{k}\left(x^{(1)}, x^{(2)}, \ldots, x^{(i-1)}, \quad x^{(i+1)}, \ldots, x^{(d-1)}, x^{(d)}\right) \\
= & f_{k}\left(x^{(1)}, x^{(2)}, \ldots, x^{(i-1)}, a, x^{(i+1)}, \ldots, x^{(d-1)}, x^{(d)}\right) .
\end{aligned}
$$

By the definition of complete monotonicity, the function $\hat{f}_{k}$ is a $(d-1)$-variate completely monotone function. Consequently, by the induction hypothesis, $\hat{f}_{k}$ is Borel measurable on $[0,1]^{d-1}$. Thus the preimage of a Borel set of $\mathbb{R}$ under $f_{k}$ consists of

- The part contained in $H_{k}$, which is the cross-product of a Borel set of $[0,1]^{d-1}$ and of a one-point set (the point $a$ in equation $(3 \cdot 2)$ ), and which consequently is $\mathcal{B}\left([0,1]^{d}\right)$-measurable.

- Possibly additionally the whole set $[0,1]^{d} \backslash H_{k}$, which is also measurable.

Thus for every $k$ the function $f_{k}$ is a measurable function from $\left([0,1]^{d}, \mathcal{B}\left([0,1]^{d}\right)\right)$ to $(\mathbb{R}, \mathcal{B}(\mathbb{R}))$.

Next we define a function $g$ by setting

$$
g(\mathbf{x})= \begin{cases}f(\mathbf{x}) & \text { for } \mathbf{x} \in D \\ m & \text { otherwise }\end{cases}
$$

where $D$ is the set from $(3 \cdot 1)$. We want to show that for every given $b \in \mathbb{R}$ the preimage of $(-\infty, b)$ under $g$ is measurable. Then, since the Borel sigma-field on $\mathbb{R}$ is generated by the collection of sets $\{(-\infty, b), b \in \mathbb{R}\}$, the function $g$ is a measurable function from $\left([0,1]^{d}, \mathcal{B}\left([0,1]^{d}\right)\right)$ to $(\mathbb{R}, \mathcal{B}(\mathbb{R}))$ (see $[\mathbf{2 1}$, Theorem 1.41]). Thus let $b \in \mathbb{R}$ be fixed, and set $B=(-\infty, b)$. If $b \leq m$, then by construction the set $g^{-1}(B)$ is the empty set (which is measurable). If $b>m$, then by construction we have

$$
\left([0,1]^{d} \backslash D\right) \subset g^{-1}(B) .
$$

Furthermore, since $f$ and $g$ coincide on $D$, we have

$$
g^{-1}(B)=f^{-1}(B) \cup\left([0,1]^{d} \backslash D\right) .
$$

Now assume that $\mathbf{x} \in D$, and that $\mathbf{x} \in f^{-1}(B)$. Then there exists a number $y \in B$ such that $y=f(\mathbf{x})$. By construction the function $f$ is continuous in $\mathbf{x}$. Note that the set $B$ is open, which implies that there exists a $\delta>0$ such that a $\delta$-neighborhood around $y$ is also contained in $B$. Accordingly, by the definition of continuity, there exists an $\varepsilon>0$ such that all elements of $[0,1]^{d}$ which are contained in an $\varepsilon$-neighborhood of $\mathbf{x}$ are mapped by $f$ into the $\delta$-neighborhood of $y$. Thus there exists an open set $N_{\mathbf{x}} \subset[0,1]^{d}$ containing $\mathbf{x}$ such that $f\left(N_{\mathbf{x}}\right) \subset B$. It is easily verified that we have

$$
\bigcup_{\mathbf{x} \in D, f(\mathbf{x}) \in B} N_{\mathbf{x}} \subset f^{-1}(B) \subset\left(\bigcup_{\mathbf{x} \in D, f(\mathbf{x}) \in B} N_{\mathbf{x}}\right) \cup\left([0,1]^{d} \backslash D\right) .
$$

Thus by $(3 \cdot 3)$ we have

$$
g^{-1}(B)=\left(\bigcup_{\mathbf{x} \in D, f(\mathbf{x}) \in B} N_{\mathbf{x}}\right) \cup\left([0,1]^{d} \backslash D\right) .
$$

The set on the right-hand side of $(3 \cdot 4)$ is the union of 
- a union of open sets (which itself is also open, and consequently Borel measurable), and of

- a countable union of hyperplanes (which also is Borel measurable).

Thus $g^{-1}(B) \in \mathcal{B}\left([0,1]^{d}\right)$, which proves that $g$ is a measurable function from $\left([0,1]^{d}, \mathcal{B}\left([0,1]^{d}\right)\right)$ to $(\mathbb{R}, \mathcal{B}(\mathbb{R}))$.

Thus we have established that all the functions $g$ and $f_{k}, k \geq 1$, are measurable. By construction we have

$$
f(\mathbf{x})=\sup _{N \geq 1} \max \left\{g(\mathbf{x}), f_{1}(\mathbf{x}), f_{2}(\mathbf{x}), \ldots, f_{N}(\mathbf{x})\right\}, \quad \mathbf{x} \in[0,1]^{d} .
$$

The supremum of measurable functions is itself measurable (see [21, Theorem 4.22]). Thus we have established that $f$ is a measurable function from $\left([0,1]^{d}, \mathcal{B}\left([0,1]^{d}\right)\right)$ to $(\mathbb{R}, \mathcal{B}(\mathbb{R}))$, which proves the theorem.

\section{Equivalence of Hardy-Krause and $\mathcal{R}^{*}$-variation}

The aim of this section is to show that $\mathcal{D}$-variation with $\mathcal{D}=\mathcal{R}^{*}$ coincides with HardyKrause variation. The following was already shown in $[\mathbf{2 0}]$.

TheOREM $4 \cdot 1([\mathbf{2 0}])$. $\mathcal{H} \mathcal{K} \cap \mathcal{V}_{\infty}\left(\mathcal{R}^{*}\right)=\mathcal{V}\left(\mathcal{R}^{*}\right)$ and $H K\left(f ;[0,1]^{d}\right)=V_{\mathcal{R}^{*}}(f)$ whenever $f \in \mathcal{V}\left(\mathcal{R}^{*}\right)$.

We shall show the following theorem.

THEOREM 4.2. Every function of bounded Hardy-Krause variation can be uniformly approximated by a sequence of simple functions from $\mathcal{S}\left(\mathcal{R}^{*}\right)$, i.e. $\mathcal{H} \mathcal{K} \subseteq \mathcal{V}_{\infty}\left(\mathcal{R}^{*}\right)$.

Combining this with Theorem $4 \cdot 1$ yields:

Corollary 4·3. We have $\mathcal{H} \mathcal{K}=\mathcal{V}\left(\mathcal{R}^{*}\right)$, and for any $f:[0,1]^{d} \rightarrow \mathbb{R}$ we have

$$
H K\left(f ;[0,1]^{d}\right)=V_{\mathcal{R}^{*}}(f) .
$$

Since the limit of a sequence of measurable functions is again measurable, we immediately obtain Theorem $3 \cdot 1$ as a corollary.

COROLlary 4.4. Every function of bounded Hardy-Krause variation is Borel measureable.

REMARK 4.5. Corollary $4 \cdot 3$ shows that $\mathcal{R}^{*}$-variation yields an alternative way of constructing Hardy-Krause variation. This is convenient in some situations, as illustrated by Corollary 4.4: Measurability is obvious from the definition of $\mathcal{R}^{*}$-variation, whereas the proof based on the classical definition is involved and relies on results of earlier papers on the points of discontinuity of a function of bounded variation. Other properties, such as the fact that if $f>\delta>0$ has bounded Hardy-Krause variation, then so does $1 / f$, are easily shown with the classical definition, but it is not clear to the authors how to obtain this fact directly from the definition of $\mathcal{R}^{*}$-variation.

Remark 4.6. Corollary $4 \cdot 3$ also shows that $\mathcal{D}$-variation is a quite general concept in the sense that the spaces $\mathcal{V}(\mathcal{D})$ of functions of bounded $\mathcal{D}$-variation are rather large and contain many interesting functions. Indeed, if $\mathcal{R}^{*} \subseteq \mathcal{D}$ then $\mathcal{H} \mathcal{K} \subseteq \mathcal{V}(\mathcal{D})$. In particular, 
the space of functions of bounded $\mathcal{K}$-variation contains $\mathcal{H} \mathcal{K}$, but is known to be strictly larger. This was not at all clear to the authors in $[\mathbf{2 0}]$.

REMARK 4.7. A Koksma-Halwka inequality for general measures on $[0,1]^{d}$, which is the main result in Götz [10] and is also stated as Theorem 1 in [2], follows directly from Corollary $4 \cdot 3$ and the Koksma-Hlawka inequality [20, Thm. 4.3].

Before we prove Theorem 4.2, we slightly extend the notation introduced in Section 2 and present an important observation on completely monotone functions. If $v \subseteq\{1, \ldots, d\}$ and $\mathbf{a}, \mathbf{b} \in[0,1]^{d}$, then $[\mathbf{a}, \mathbf{b}]^{v}$ denotes the box

$$
[\mathbf{a}, \mathbf{b}]^{v}=\left\{\mathbf{x} \in \mathbb{R}^{d} \mid \forall i \in v: a_{i} \leq x_{i} \leq b_{i}, \forall i \notin v: a_{i} \leq x_{i}<b_{i}\right\} .
$$

If $a_{i}=b_{i}$ for some $i \notin v$, then $[\mathbf{a}, \mathbf{b}]^{v}$ should be interpreted as the empty set. The set of axis-parallel rectangles containing $\mathbf{0}$ is then given by

$$
\mathcal{R}^{*}=\left\{[\mathbf{0}, \mathbf{a}]^{v} \mid \mathbf{a} \in[0,1]^{d}, v \subseteq\{1, \ldots, d\}\right\} .
$$

Given a ladder $\mathcal{Y}$, we define a partial ordering of the pairs $(\mathbf{y}, v)$ with $\mathbf{y} \in \bigcup_{u \subseteq\{1, \ldots, d\}} \mathcal{Y}_{u}$ and $v \subseteq\{1, \ldots, d\}$ by declaring $(\mathbf{y}, v) \leq(\mathbf{z}, w)$ if $\mathbf{y} \leq \mathbf{z}$ and $\mathbf{y} \neq \mathbf{z}$ or if $\mathbf{y}=\mathbf{z}$ and $v \subseteq w$. We denote by $C(\mathbf{y}, v)$ the face

$$
[\mathbf{0}, \mathbf{y}]^{v} \backslash \bigcup_{(\mathbf{z}, w)<(\mathbf{y}, v)}[\mathbf{0}, \mathbf{z}]^{w} .
$$

Intuitively, $C(\mathbf{y}, v)$ denotes the $d-|v|$-dimensional face of the subrectangle formed by the ladder whose maximal vertex is $\mathbf{y}$ and whose spanning edges specified by $v$ have maximal vertex y; see Figure 1 (left).
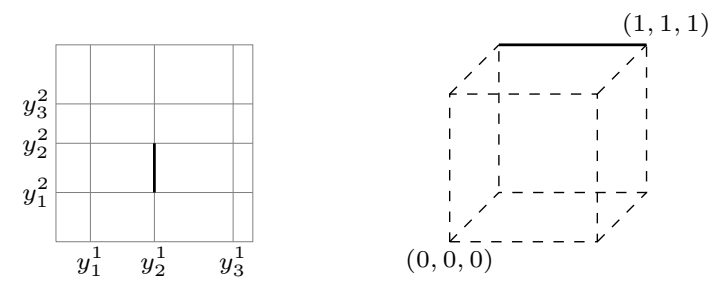

Fig. 1. Left: A grid ladder (gray) and the face $C\left(\left(y_{2}^{1}, y_{2}^{2}\right),\{1\}\right)$ (bold). Right: The cube $[0,1]^{3}$ (dashed) and the face $F_{1}$ (bold) - this will be defined and used at the end of this section.

Moreover, for $\mathbf{x}, \mathbf{y}, \mathbf{a} \in[0,1]^{d}$ and $i, j \in\{1, \ldots, d\}$ with $i \neq j$ we write $\mathbf{y}^{i}: \mathbf{x}^{j}: \mathbf{a}^{-i \cup j}$ for the point with $i$-th coordinate $y_{i}, j$-th coordinate $x_{j}$, and all other coordinates equal to those of $\mathbf{a}$.

Lemma 4.8. Let $f$ be a completely monotone function and let $\mathbf{x}, \mathbf{a} \in[0,1]^{d}$ and $i, j \in$ $\{1, \ldots, d\}, i \neq j$. Then

$$
\begin{aligned}
\left|f\left(\mathbf{x}^{j}: \mathbf{a}^{-j}\right)-f(\mathbf{a})\right| & \leq\left|f\left(\mathbf{1}^{i}: \mathbf{x}^{j}: \mathbf{a}^{-i \cup j}\right)-f\left(\mathbf{1}^{i}: \mathbf{a}^{-i}\right)\right| \\
& \leq\left|f\left(\mathbf{x}^{j}: \mathbf{1}^{-j}\right)-f\left(\mathbf{a}^{j}: \mathbf{1}^{-j}\right)\right| .
\end{aligned}
$$

Proof. We use the complete monotonicity of $f$ restricted to the 2-dimensional plane $\left\{\mathbf{z} \in[0,1]^{d}: \mathbf{z}^{-i \cup j}=\mathbf{a}^{-i \cup j}\right\}$. If $a_{j} \leq x_{j}$, we get

$$
0 \leq f(\mathbf{a})+f\left(\mathbf{1}^{i}: \mathbf{x}^{j}: \mathbf{a}^{-i \cup j}\right)-f\left(\mathbf{x}^{j}: \mathbf{a}^{-j}\right)-f\left(\mathbf{1}^{i}: \mathbf{a}^{-i}\right),
$$


which, together with the monotonicity on 1-dimensional spaces, yields

$$
0 \leq f\left(\mathbf{x}^{j}: \mathbf{a}^{-j}\right)-f(\mathbf{a}) \leq f\left(\mathbf{1}^{i}: \mathbf{x}^{j}: \mathbf{a}^{-i \cup j}\right)-f\left(\mathbf{1}^{i}: \mathbf{a}^{-i}\right) .
$$

If $a_{j} \geq x_{j}$, all inequalities are reversed, so in both cases, we can deduce

$$
\left|f\left(\mathbf{x}^{j}: \mathbf{a}^{-j}\right)-f(\mathbf{a})\right| \leq\left|f\left(\mathbf{1}^{i}: \mathbf{x}^{j}: \mathbf{a}^{-i \cup j}\right)-f\left(\mathbf{1}^{i}: \mathbf{a}^{-i}\right)\right| .
$$

The second inequality follows by repeated use of the first one.

Proof of Theorem $4 \cdot 2$. By Lemma $2 \cdot 1$, we may assume that $f$ is completely monotone. We prove the theorem in two steps. We first study the case $d=1$, and then we apply this result to approximate functions on $[0,1]^{d}$ for general $d$ as well.

First let $d=1$. A completely monotone function $f:[0,1] \rightarrow \mathbb{R}$ is a bounded increasing function. In particular, we may choose a simple function $g_{n}$ with $\left|f-g_{n}\right|_{\infty} \leq 1 / n$. One can construct $g_{n}$ as follows: Choose a partion inf $f=t_{1}<\cdots<t_{N+1}=\sup f$ of the interval $[\inf f, \sup f]$ such that $\left|t_{l}-t_{l+1}\right| \leq 1 / n$ for all $l$. Then choose $y_{l} \in f^{-1}\left(t_{l}\right)$ if such a point exists. Otherwise $f^{-1}\left(t_{l}\right)=\emptyset$, which means that $f$ has a jump from values smaller than $t_{l}$ to values larger than $t_{l}$ at some point. We denote this point by $y_{l}$. We may take $y_{1}=0, y_{N+1}=1$. (Some of the $y_{l}$ may be equal. In this case, we throw away multiple points so that $y_{l}<y_{l+1}$ for all $l$.)

On each interval $\left(y_{l}, y_{l+1}\right), f$ is increasing and its function values lie in $\left[t_{l}, t_{l+1}\right]$. Therefore, if we choose a point $z_{l} \in\left(y_{l}, y_{l+1}\right)$, then for any $x \in\left(y_{l}, y_{l+1}\right)$ we have $\left|f(x)-f\left(z_{l}\right)\right| \leq$ $\left|t_{l+1}-t_{l}\right| \leq 1 / n$. Thus, if we define $g_{n}$ such that $g_{n}(x)=f\left(z_{l}\right)$ for $x \in\left(y_{l}, y_{l+1}\right)$ and $g_{n}(x)=f\left(y_{l}\right)$ for $x=y_{l}$, then $\left|g_{n}-f\right|_{\infty} \leq 1 / n$.

We need to show that $g_{n} \in \mathcal{S}\left(\mathcal{R}^{*}\right)$. To achieve this, we define a new step function $f_{n}$ as a sum of contributions from the half open intervals $\left[0, y_{l}\right)$ and the closed intervals $\left[0, y_{l}\right]$. The contribution to $f_{n}$ from the half open intervals are $f\left(z_{l-1}\right)-f\left(y_{l}\right)$, and $f\left(y_{l}\right)-f\left(z_{l}\right)$ from the closed intervals (with the exception of the interval $[0,1]$ which contributes $f(1)$ ). In this way, $f_{n}(x)$ is written as a telescoping sum and evaluates to either $f\left(y_{l}\right)$ or $f\left(z_{l}\right)$ depending on whether $x=y_{l}$ or $x \in\left(y_{l}, y_{l+1}\right)$. Thus $f_{n}(x)$ agrees with $g_{n}(x)$ for all $x \in[0,1]$. Using the heavy notation introduced above, we can write $f_{n}$ as

$$
f_{n}=\sum_{l=1}^{N+1} \sum_{v \subseteq\{1\}} \alpha_{l, v} \mathbb{1}_{\left[0, y_{l}\right]^{v}} .
$$

Here $\alpha_{N+1,\{1\}}=f(1)$, and for $(l, v)<(N+1,\{1\})$, we define $\alpha_{l, v}$ as follows: Let $z_{l,\{1\}}=y_{l}$ and $z_{l, \emptyset}=z_{l-1}$. Let $\mathcal{Y}$ be the ladder formed by $y_{1}, \ldots, y_{N}$ and $\tilde{\mathcal{Y}}$ the ladder formed by the $z_{l, v}$ with $(l, v)<(N+1,\{1\})$. Then

$$
\alpha_{l, v}=-\Delta\left(f ; z_{l, v},\left(\tilde{z}_{l, v}\right)_{+}\right)=f\left(z_{l, v}\right)-f\left(\left(\tilde{z}_{l, v}\right)_{+}\right) .
$$

Here $\tilde{y}_{+}$indicates that the successor of $y$ is computed with respect to the ladder $\tilde{\mathcal{Y}}$. With this definition, $f_{n}(x)$ agrees with $g_{n}(x)$ for all $x \in[0,1]$, which concludes the argument in the one-dimensional case.

This idea can be extended to the multi-dimensional case by replacing the half open intervals by partially open axis aligned boxes to which we attach alternating sums of 
function values. To see this, let $d>1$ and consider a completely monotone function $f$ on $[0,1]^{d}$. Let $f^{i}, i=1, \ldots, d$, denote the restriction of $f$ to the 1 -dimensional face $F_{i}=\left\{\mathbf{x} \in[0,1]^{d} \mid \mathbf{x}^{-i}=\mathbf{1}^{-i}\right\}$ of $[0,1]^{d}$; see Figure 1 (right) for an illustration of $F_{i}$. We apply the result for $d=1$ to each $f^{i}$ and define ladders $\mathcal{Y}^{i}$ and $\tilde{\mathcal{Y}}^{i}$ consisting of points $\mathbf{y}_{l}^{i}$ and $\mathbf{z}_{l, v}^{i}$, respectively, such that we have an approximation $f_{n}^{i}$ of the form (4.1), i.e.

$$
f_{n}^{i}=\sum_{l=1}^{N_{i}+1} \sum_{v \subseteq\{1\}} \alpha_{l, v}^{i} \mathbb{1}_{\left[\mathbf{0}, \mathbf{y}_{l}^{i}\right]^{v}}
$$

and $\left|f^{i}-f_{n}^{i}\right|_{\infty} \leq 1 / n$.

Form the ladders $\mathcal{Y}=\prod_{i} \mathcal{Y}^{i}$ and $\tilde{\mathcal{Y}}=\prod_{i} \tilde{\mathcal{Y}}^{i}$. For $\mathbf{y}=\left(y_{l_{1}}^{1}, \ldots, y_{l_{d}}^{d}\right) \in \mathcal{Y}_{u}$, let $\mathbf{z}_{y, v}$ be the point whose $i$-th coordinate is $z_{l_{i},\{1\}}^{i}=y_{l_{i}}^{i}$ if $i \in v$, and $z_{l_{i}, \emptyset}^{i}$ otherwise. Then $\mathbf{z}_{y, v} \in C(\mathbf{y}, v)$. Consider the simple function

$$
f_{n}(\mathbf{x})=\sum_{u \subseteq\{1, \ldots, d\}} \sum_{\mathbf{y} \in \mathcal{Y}_{u}} \sum_{v \subseteq\{1, \ldots, d\}} \alpha_{u, \mathbf{y}, v} \mathbb{1}_{[\mathbf{0}, \mathbf{y}]^{v}}(\mathbf{x})
$$

where

$$
\alpha_{u, \mathbf{y}, v}=(-1)^{|u \cup(-v)|} \Delta_{u \cup(-v)}\left(f, \mathbf{z}_{y, v}, \tilde{\mathbf{z}}_{y, v}^{+}\right) .
$$

Here $\tilde{\mathbf{y}}^{+}$is the successor of $\mathbf{y}$ in its ladder.

Theorem 4.2 is proved if we can show that $\left|f_{n}-f\right|_{\infty} \leq d / n$. Note that $f_{n}$ is constantly equal to $f\left(\mathbf{z}_{y_{0}, v_{0}}\right)$ on each $C\left(\mathbf{y}_{0}, v_{0}\right)$ because for $\mathbf{x} \in C\left(\mathbf{y}_{0}, v_{0}\right)$ we have

$$
\begin{aligned}
f_{n}(\mathbf{x}) & =\sum_{u \subseteq\{1, \ldots, d\}} \sum_{\mathbf{y} \in \mathcal{Y}_{u}} \sum_{v \subseteq\{1, \ldots, d\}} \alpha_{u, \mathbf{y}, v} \mathbb{1}_{[\mathbf{0}, \mathbf{y}]^{v}}(\mathbf{x}) \\
& =\sum_{u \subseteq\{1, \ldots, d\}} \sum_{\mathbf{y} \in \mathcal{Y}_{u}} \sum_{v \subseteq\{1, \ldots, d\}}(-1)^{|u \cup(-v)|} \Delta_{u \cup(-v)}\left(f, \mathbf{z}_{y, v}, \tilde{\mathbf{z}}_{y, v}^{+}\right) \mathbb{1}_{\left[\mathbf{0}, \mathbf{z}_{y, v}\right]}\left(\mathbf{z}_{y_{0}, v_{0}}\right) \\
& =\sum_{u \subseteq\{1, \ldots, d\}} \sum_{\mathbf{z} \in \tilde{\mathcal{Y}}_{u}}(-1)^{|u|} \Delta_{u}\left(f, \mathbf{z}, \tilde{\mathbf{z}}_{+}\right) \mathbb{1}_{[\mathbf{0}, \mathbf{z}]}\left(\mathbf{z}_{y_{0}, v_{0}}\right) \\
& =f\left(\mathbf{z}_{y_{0}, v_{0}}\right),
\end{aligned}
$$

where the last equality uses [19, Proposition 6]. Observe also that $f_{n}=f_{n}^{i}$ on each of the faces $F_{i}$. Let $\mathbf{x} \in C(\mathbf{y}, v)$ be given and write $\mathbf{q}=\mathbf{z}_{y, v}$. Then

$$
\begin{aligned}
\left|f(\mathbf{x})-f_{n}(\mathbf{x})\right| & =|f(\mathbf{x})-f(\mathbf{q})| \\
& \leq \sum_{i=1}^{d}\left|f\left(x_{1}, \ldots, x_{i}, q_{i+1}, \ldots, q_{d}\right)-f\left(x_{1}, \ldots, x_{i-1}, q_{i}, \ldots, q_{d}\right)\right| \\
& \leq \sum_{i=1}^{d}\left|f\left(1, \ldots, 1, x_{i}, 1 \ldots 1\right)-f\left(1, \ldots, 1, q_{i}, 1 \ldots, 1\right)\right| \\
& =\sum_{i=1}^{d}\left|f^{i}\left(x_{i}\right)-f_{n}^{i}\left(q_{i}\right)\right| \\
& \leq d / n,
\end{aligned}
$$

where the second inequality follows from Lemma $4 \cdot 8$ and the second equality used that $f_{n}^{i}\left(\mathbf{z}_{l, v}^{i}\right)=f\left(\mathbf{z}_{l, v}^{i}\right)$ by construction. 
5. Algebraic structure of $\mathcal{V}(\mathcal{D})$

Finally, we consider the algebraic structure of the function space $\mathcal{V}(\mathcal{D})$. We will assume throughout this section that $\mathcal{D}$ is closed under intersection, i.e. $D_{1}, D_{2} \in \mathcal{D}$ implies $D_{1} \cap D_{2} \in \mathcal{D}$.

\subsection{Algebraic Structure of $\mathcal{V}(\mathcal{D})$}

Analogous to [4] we define for $f \in \mathcal{V}(\mathcal{D})$ and $\sigma>0$

$$
\|f\|=\|f\|_{\infty}+\sigma V_{\mathcal{D}}(f),
$$

which is a norm on $\mathcal{V}(\mathcal{D})$. In the following we show that for $\sigma \geq 3,(\mathcal{V}(\mathcal{D}),\|\cdot\|)$ is a commutative Banach algebra with respect to pointwise multiplication.

Lemma $5 \cdot 1$. The norm $\|\cdot\|$ is complete.

Proof. Let $\left(f_{i}\right)$ be a Cauchy sequence in $(\mathcal{V}(\mathcal{D}),\|\cdot\|)$. Then $\left\|f_{i}-f_{j}\right\|<\varepsilon$ implies that

$$
\left\|f_{i}-f_{j}\right\|_{\infty}<\varepsilon \quad \text { and } \quad \sigma V_{\mathcal{D}}\left(f_{i}-f_{j}\right)<\varepsilon,
$$

because both summands are nonnegative, since $\sigma>0,\|\cdot\|_{\infty}$ is a norm and $V_{\mathcal{D}}$ is a seminorm. This implies that $\left(f_{i}\right)$ is a Cauchy sequence with respect to the supremum norm and hence converges uniformly to some $f \in \mathcal{V}_{\infty}(\mathcal{D})$. Now choose a subsequence $i_{k}$ such that $V_{\mathcal{D}}\left(f_{i_{k}}-f_{i_{k}+j}\right)<1 / 2^{k}$ for all $j>0$. Then $\left(f_{i_{k}}\right)_{k \geq 1}$ is also a Cauchy sequence with respect to the supremum norm and converges uniformly to $f$. The semi-continuity (see [20, Proposition 3.6]) yields that

$$
V_{\mathcal{D}}\left(f-f_{i}\right) \leq \liminf _{k} V_{\mathcal{D}}\left(f_{i_{k}}-f_{i}\right),
$$

which is smaller than some given $\varepsilon>0$ if $i$ is sufficiently large. Thus, $f \in \mathcal{V}(\mathcal{D})$.

The next thing is to show that the Banach space norm is submultiplicative.

Lemma 5.2. Assume $\mathcal{D}$ is closed under intersections. Let $f, g \in(\mathcal{V}(\mathcal{D}),\|\cdot\|)$ and let $\sigma \geq 3$. Then $\|f g\| \leq\|f\|\|g\|$.

Proof. This follows via a direct calculation from $(2 \cdot 1)$ :

$$
\begin{aligned}
\|f g\| & =\|f g\|_{\infty}+\sigma V_{\mathcal{D}}(f g) \\
& \leq\|f g\|_{\infty}+\sigma \inf |f| V_{\mathcal{D}}(g)+\sigma \inf |g| V_{\mathcal{D}}(f)+3 \sigma V_{\mathcal{D}}(f) V_{\mathcal{D}}(g) \\
& \leq\|f\|_{\infty}\|g\|_{\infty}+\sigma\|f\|_{\infty} V_{\mathcal{D}}(g)+\sigma\|g\|_{\infty} V_{\mathcal{D}}(f)+\sigma^{2} V_{\mathcal{D}}(f) \mathcal{V}(\mathcal{D})(g) \\
& =\|f\|\|g\| .
\end{aligned}
$$

TheOREM 5.3. If $\mathcal{D}$ is closed under intersections and $\sigma \geq 3$, then $(\mathcal{V}(\mathcal{D}),\|\cdot\|)$ is a commutative Banach algebra with respect to pointwise multiplication.

Proof. By Proposition $2 \cdot 3$ and Lemma $5 \cdot 1,(\mathcal{V}(\mathcal{D}),+,\|\cdot\|)$ is a complete, normed vector space. Moreover, $(\mathcal{V}(\mathcal{D}),+, \cdot)$ is an associative and commutative $\mathbb{R}$-algebra with respect to pointwise multiplication. Finally, by Lemma $5 \cdot 2$, the norm is also submultiplicative. 


\section{5·2. Further Properties}

In [5] the maximal ideal space of the Banach algebra of functions of bounded Hardy Krause variation was determined. This result is based on two key observations, [5, Lemma $6]$ and [5, Proposition 3]. In the following we briefly discuss the difficulties in generalising these results to our new notion.

We start by recalling the results of [5]. To state the first one, we introduce the function

$$
\operatorname{sgn}(x)= \begin{cases}1 & \text { if } x>0 \\ 0 & \text { if } x=0 \\ -1 & \text { if } x<0\end{cases}
$$

Lemma $5 \cdot 4$ (Blümlinger, [5]). Let $f:[0,1]^{d} \rightarrow \mathbb{R}$ be of bounded Hardy-Krause variation. Let $\left(\mathbf{x}^{(n)}\right)_{n \geq 1}$ be a sequence in $[0,1]^{d}$ converging to some $\mathbf{x}$ and having the property that $\operatorname{sgn}\left(x_{i}^{(n)}-x_{i}\right)$ depends only on $i$, but not on $n$. Then $\lim _{n \rightarrow \infty} f\left(\mathbf{x}^{(n)}\right)$ exists.

This Lemma was used to prove the following classification of the maximal ideals in $\mathcal{H K}$.

TheOREm 5.5 (Blümlinger, [5]). The maximal ideals in $\mathcal{H K}$ are in one-to-one correspondance with the set of pairs $(\mathbf{x}, \varepsilon) \in[0,1]^{d} \times\{-1,0,1\}^{d}$ satisfying $0 \leq \varepsilon_{i}$ if $x_{i}=0$ and $\varepsilon_{i} \leq 0$ if $x_{i}=1$. The pair $(\mathbf{x}, \varepsilon)$ corresponds to the ideal consisting of functions $f \in \mathcal{H} \mathcal{K}$ such that $\lim _{\mathbf{x}^{(n)} \rightarrow \mathbf{x}} f\left(\mathbf{x}^{(n)}\right)=0$ for any sequence $\left(\mathbf{x}^{(n)}\right)_{n \geq 0}$ converging to $\mathbf{x}$ and having $\operatorname{sgn}\left(x_{i}^{(n)}-x_{i}\right)=\varepsilon_{i}$.

Lemma $5 \cdot 4$ does not hold in general for functions of bounded $\mathcal{D}$-variation when $\mathcal{D} \neq \mathcal{R}^{*}$, as the following example shows.

EXAmple 5.6. The function $f:[0,1]^{2} \rightarrow \mathbb{R}$ given by $f(\mathbf{x})=\mathbb{1}_{x_{1}>x_{2}}$ has bounded $\mathcal{K}$ variation. However, the sequence $\mathbf{x}^{(n)}=\left(1 / 2+3 / n, 1 / 2+\left(2+(-1)^{n}\right) / n\right)$ converges to $\mathbf{x}=(1 / 2,1 / 2)$ and $\operatorname{sgn}\left(x_{i}^{(n)}-x_{i}\right)=1$ for all $n$, but $f\left(\mathbf{x}^{(n)}\right)$ alternates between 0 and 1 , so $\lim _{n \rightarrow \infty} f\left(\mathbf{x}^{(n)}\right)$ does not exist.

Instead, the following weaker version of Lemma $5 \cdot 4$ holds for $\mathcal{K}$-variation. For $\mathbf{x} \in[0,1]^{d}$ and a unit vector $\mathbf{u} \in \mathbb{S}^{d-1}$, we define $L_{\mathbf{x}, \mathbf{u}}=\{\mathbf{x}+t \mathbf{u} \mid t>0\}$ to be the open half line starting from $\mathbf{x}$ and spanned by $\mathbf{u}$.

Proposition 5.7. Suppose $f:[0,1]^{d} \rightarrow \mathbb{R}$ has bounded $\mathcal{K}$-variation. Let $\mathbf{x} \in[0,1]^{d}$ and $\mathbf{u} \in \mathbb{S}^{d-1}$ and let $\mathbf{x}^{(n)}$ be a sequence contained in $L_{\mathbf{x}, \mathbf{u}}$ converging to $\mathbf{x}$. Then $\lim _{n \rightarrow \infty} f\left(\mathbf{x}^{(n)}\right)$ exists.

Proof. Let $K \subseteq[0,1]^{d}$ be a convex set. Then there is an $\varepsilon>0$ such that either $B_{\varepsilon}(\mathbf{x}) \cap L_{\mathbf{x}, \mathbf{u}} \subseteq K$ or $B_{\varepsilon}(\mathbf{x}) \cap L_{\mathbf{x}, \mathbf{u}} \subseteq \mathbb{R}^{d} \backslash K$. This is obvious if $K \cap L_{\mathbf{x}, \mathbf{u}}=\emptyset$. Otherwise, $K \cap L_{\mathbf{x}, \mathbf{u}}$ contains a point $\mathbf{y}$. The open line segment between $\mathbf{x}$ and $\mathbf{y}$ is either contained in $K \cap L_{\mathbf{x}, \mathbf{u}}$ or it contains a point $\mathbf{y}^{\prime}=\lambda \mathbf{x}+(1-\lambda) \mathbf{y}$ with $\lambda \in(0,1)$ that does not belong to $K$. By convexity, the line segment between $\mathbf{x}$ and $\mathbf{y}^{\prime}$ cannot contain any point from $K$. This yields the proposition for $f=\mathbb{1}_{K}$, and hence also when $f$ is a simple function.

Let $\mathbf{x}^{(n)}$ be a sequence in $L_{\mathbf{x}, \mathbf{u}}$ converging to $\mathbf{x}$ and let $f$ be a general function of bounded $\mathcal{K}$-variation. We must show that $f\left(\mathbf{x}^{(n)}\right)$ is a Cauchy sequence. Let $\varepsilon>0$ be given and 
choose a simple function $g$ with $|f-g|_{\infty} \leq \varepsilon / 3$. Then $\left|f\left(\mathbf{x}^{(n)}\right)-f\left(\mathbf{x}^{(m)}\right)\right| \leq \mid g\left(\mathbf{x}^{(n)}\right)-$ $g\left(\mathbf{x}^{(m)}\right) \mid+2 \varepsilon / 3$. Choose $N$ such that $\left|g\left(\mathbf{x}^{(n)}\right)-g\left(\mathbf{x}^{(m)}\right)\right| \leq \varepsilon / 3$ for all $m, n \geq N$. Then $\left|f\left(\mathbf{x}^{(n)}\right)-f\left(\mathbf{x}^{(m)}\right)\right| \leq \varepsilon$ for all $m, n \geq N$.

The obvious generalization of Theorem $5 \cdot 5$ would be that the maximal ideals consist of functions whose limits along a fixed line segment $L_{\mathbf{x}, \mathbf{u}}$ vanish. However, we have not been able to show this. An important ingredient in the proof of Theorem 5.5 is Proposition 3 of $[5]$ :

Proposition 5.8 (Blümlinger, [5]). If $f \in \mathcal{H} \mathcal{K}$ and there is a $\delta>0$ such that $|f| \geq \delta$, then $1 / f \in \mathcal{H} \mathcal{K}$.

While it is relatively simple to see this from the definition of Hardy-Krause variation, it is not obvious to the authors whether a similar statement can be shown for functions of bounded $\mathcal{K}$-variation. It is true, however, for functions of bounded generalised Harman variation; see [20, Corollary 3.17].

\section{Acknowlegdements}

The first author is supported by a Schrödinger scholarship of the Austrian Science Fund (FWF), and by FWF project I 1751-N26. The first and fourth author are supported by FWF projects F 5507 and 5509, which are parts of the Special Research Program Quasi-Monte Carlo Methods: Theory and Applications. The third author is supported by the Centre for Stochastic Geometry and Advanced Bioimaging, funded by the Villum Foundation.

Finally, we would especially like to thank the referee for a very careful study of our manuscript that helped to remove various inaccuracies in an earlier version of this paper.

\section{REFERENCES}

[1] C. R. Adams, J. A. Clarkson, Properties of functions $f(x, y)$ of bounded variation, Trans. Amer. Math. Soc. 36 (1934), 711-730.

[2] C. Aistleitner, J. Dick, Functions of bounded variation, signed measures and a general Koksma-Hlawka inequality, Acta Arith 167 (2015), 143-171.

[3] P. Antosik, Study of the continuity of a function of many variables (Russian), Prace Mat. 10 (1966), 101-104.

[4] M. Blümlinger, R. F. Tichy, Topological Algebras of Functions of Bounded Variation I, Manuscripta Math. 65 (1989), 245-255.

[5] M. Blümlinger, Topological Algebras of Functions of Bounded Variation II, Manuscripta Math. 65 (1989), 377-384.

[6] L. Brandolini, L. Colzani, G. Gigante, G. Travaglini, On the Koksma-Hlawka inequality, J. Complexity 29 (2013), 158-172.

[7] L. Brandolini, L. Colzani, G. Gigante, G. Travaglini, A Koksma-Hlawka inequality for simplices, Trends in harmonic analysis, 33-46, Springer INdAM Ser. 3, Springer, Milan, 2013.

[8] J. A. Clarkson, C. R. Adams, On definitions of bounded variation for functions of two variables, Trans. Amer. Math. Soc. 35 (1933), 824-854.

[9] M. Drmota, R.F. Tichy, Sequences, discrepancies and applications. Lecture Notes in Mathematics, 1651. Springer-Verlag, Berlin, 1997.

[10] M. Götz, Discrepancy and the error in integration, Monatsh. Math. 136 (2002), no. 2, 99-121.

[11] G. H. Hardy, On double Fourier series, and especially those which represent the double zeta-function with real and incommensurable parameters, Quart. J. Math. (1) 37 (1906), $53-79$.

[12] G. Harman, Variations on the Koksma-Hlawka inequality, Unif. Distrib. Theory 5 (2010), $65-78$. 
[13] E. Hlawka, Funktionen von beschränkter Variation in der Theorie der Gleichverteilung, Ann. Math. Pura Appl. 54 (1961), 325-333.

[14] D. Idczak, Functions of several variables of finite variation and their differentiability, Ann. Polon. Math. 60 (1994), no. 1, 47-56.

[15] J. F. Koksma, A general theorem from the theory of uniform distribution modulo 1, Mathematica B (Zutphen) 11 (1942), 7-11.

[16] J. M. Krause, Fouriersche Reihen mit zwei veränderlichen Grössen, Ber. Sächs. Akad. Wiss. Leipzig 55 (1903) 164-197.

[17] L. Kuipers, H. Niederreiter, Uniform distribution of sequences. Wiley-Interscience (John Wiley \& Sons), New York-London-Sydney, 1974.

[18] A. S. Leonov, Remarks on the total variation of functions of several variables and on a multidimensional analogue of Helly's choice principle. (in Russian) Mat. Zametki 63 (1998), 69-80.

[19] A. B. Owen, Multidimensional variation for quasi-Monte Carlo, Contemporary multivariate analysis and design of experiments, 49-74, Ser. Biostat. 2, World Sci. Publ., Hackensack, NJ, 2005.

[20] F. Pausinger, A. M. Svane, A Koksma-Hlawka inequality for general discrepancy systems, J. Complexity 31 (2015), 773-797.

[21] J. Yeh, Real analysis. Theory of measure and integration. Second edition. World Scientific Publishing Co. Pte. Ltd., Hackensack, NJ, 2006.

[22] W. H. Young, G. Young, On the Discontinuties of Monotone Functions of Several Variables, Proc. London Math. Soc. (2) 22 (1923), 124-142. 